\title{
Diamond coated silicon field emitter array
}

\author{
S. Albin, ${ }^{\text {a) }}$ W. Fu, A. Varghese, and A. C. Lavarias \\ Microelectronics Research Laboratory, Old Dominion University, Norfolk, Virginia 23529 \\ G. R. Myneni \\ Accelerator Development, Thomas Jefferson National Accelerator Facility, Newport News, Virginia 23606
}

(Received 19 October 1998; accepted 21 January 1999)

\begin{abstract}
Diamond coated silicon tip arrays, with and without a self-aligned gate, were fabricated, and current-voltage characteristics of 400 tips were measured. Diamond films were grown uniformly on Si tips using microwave plasma after nucleation with $10 \mathrm{~nm}$ diamond suspension and substrate bias. An emission current of $57 \mu \mathrm{A}$ was obtained at $5 \mathrm{~V}$ from the ungated array tips separated from an anode at $2 \mu \mathrm{m}$. In the case of the gated arrays with $1.5 \mu \mathrm{m}$ aperture, an emission current of $3.4 \mu \mathrm{A}$ was measured at a gate voltage of $80 \mathrm{~V}$ for an anode separation of $200 \mu \mathrm{m}$. The turn-on voltages for these two types of devices were 0.2 and $40 \mathrm{~V}$, respectively. Diamond coated Si tip arrays have potential applications in field emission based low voltage vacuum electronic devices and microsensors. (C) 1999 American Vacuum Society. [S0734-2101(99)05804-2]
\end{abstract}

\section{INTRODUCTION}

Various field emitter arrays have been developed for flat panel displays, cold cathode electron sources, pressure sensors, and vacuum electronics devices. Two frequently employed device structures are the Spindt type and the Betsui type using metal and silicon, respectively. ${ }^{1,2}$ While both types have been successfully used in many applications, emitter performance is found to be sensitive to the vacuum environment. The critical field for stable emission needs to be improved in order to achieve large-scale commercial applications. Diamond thin film is a suitable material for field emitter arrays. Owing to its unique material properties, such as negative electron affinity, high thermal conductivity, hardness, and chemical inertness, diamond coated field emitter arrays could be operated at reduced field producing more stable and higher emission current. The high vacuum requirement for reliable operation could also be relaxed.

Both diamond and diamond-like carbon (DLC) films have been grown on flat substrates and sharpened silicon tip arrays. $^{3-5}$ Although low turn-on field for electron emission has been achieved, emission from a flat surface is nonuniform due to lack of control on the emission sites. ${ }^{6}$ Sharpened emitter arrays have more control on the emission site due to the field enhancement at the tips. ${ }^{7}$ Nucleation of diamond on the delicate emitter tips cannot be achieved with conventional diamond paste abrasion. Bias enhanced nucleation does not provide the desired nucleation density for a thin, uniform coating on the emitter tip arrays. Generally, a thick diamond coating is needed to coat the emitter array uniformly, reducing the sharpness of the tip and blocking the electron transport to the surface. ${ }^{8}$ Thin DLC coating could be used, but its emission is not stable and tends to decay rapidly with time. ${ }^{9}$ In this article, we present details of fabrication of micromachined field emitter arrays with and without a selfaligned gate using wet chemical etching. A novel diamond nucleation method is developed to grow uniform thin dia-

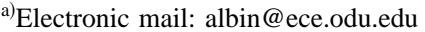

mond coating for the field emitter arrays, and emission properties are studied by current-voltage $(I-V)$ measurements.

\section{EXPERIMENTS}

\section{A. Silicon tip array fabrication}

$\langle 100\rangle n$-type silicon samples of $5-10 \Omega$-cm resistivity were used as the substrate for the field emitter arrays, and a $0.2 \mu \mathrm{m}$ thick thermal oxide was grown by the wet oxidation method. A $20 \times 20$ array of oxide consisting of $10 \mu \mathrm{m}$ squares at $20 \mu \mathrm{m}$ pitch was patterned by photolithography and buffered hydrofluoric (HF) acid etching. Orientation dependent wet chemical etching was carried out in $40 \%$ tetramethylammonium hydroxide (TMAH) at $90{ }^{\circ} \mathrm{C}$ to form a uniform Si tip array under the oxide patterns. The silicon etch rate was $0.5 \mu \mathrm{m} / \mathrm{min}$. $\mathrm{A} \mathrm{SiO}_{2}$ spacer pad was left around the array to prevent the anode from touching the tips during $I-V$ measurement. The etching was continued until the undercut of the silicon beneath the oxide square patterns was complete to form sharp tips. A thermal oxidation process further sharpened these tips. For the self-aligned gated array, $1 \mu \mathrm{m}$ oxide was grown followed by deposition of $0.2 \mu \mathrm{m}$ tungsten film using rf sputtering. A photoresist etch back process similar to the method used by Zhu et al. was employed to expose the gate metal. ${ }^{10}$ The self-aligned gated structure was formed by removing the exposed tungsten in concentrated $\mathrm{H}_{2} \mathrm{O}_{2}$ and the oxide in buffered HF.

\section{B. Diamond nucleation and growth}

A high nucleation density was achieved by incorporating nanocrystalline diamond on the silicon tip array and by applying a negative bias during the film growth. Prior to nucleation, the natural oxide on the silicon tip array was removed by dilute HF. The sample was dipped into $10 \mathrm{~nm}$ ultrafine diamond slurry, which was uniformly adsorbed onto the silicon surface. After nucleation, the samples were rinsed in de-ionized water and dried. Diamond growth on the silicon tip array was conducted using a microwave plasma chemical 


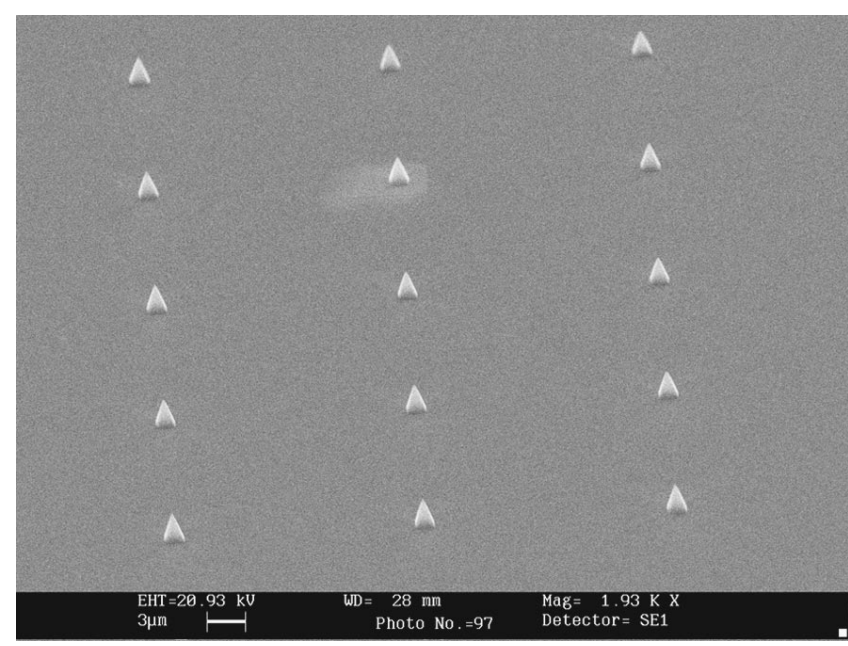

FIG. 1. Silicon tip array produced by micromachining. The scale bar corresponds to $3 \mu \mathrm{m}$.

vapor deposition system in a mixture of $1 \%$ methane in hydrogen at 35 Torr. The substrate temperature was $750{ }^{\circ} \mathrm{C}$ and the microwave power was $1 \mathrm{~kW}$. A $150 \mathrm{~V}$ negative bias was applied to the substrate to enhance nucleation during the initial $15 \mathrm{~min}$, and further growth was conducted for $15 \mathrm{~min}$ without bias.

\section{Emission I- $V$ measurement}

The diamond coated field emitter arrays were characterized by scanning electron microscopy (SEM) and $I-V$ measurement in a high vacuum chamber at $10^{-6}$ Torr. An anode consisting of an $n$-type polished silicon piece with $1 \mu \mathrm{m}$ thermal oxide was placed on top of the diamond field emitter array. The oxide at the center of the anode was removed to expose the silicon. Electrical contacts were made by applying conductive silver epoxy on the back side of the anode and the emitter array. $I-V$ measurements were conducted with a high voltage source (Keithley 273) and an electrometer (Keithley 683) interfaced to a computer through a general purpose interface bus (GPIB) card for data collection and analysis. For the gated sample, a built-in voltage source from the electrometer was connected to bias the gate, and the anode was positioned $200 \mu \mathrm{m}$ above the emitter surface with a collection voltage of $400 \mathrm{~V}$.

\section{RESULTS AND DISCUSSION}

\section{A. Wet etching}

TMAH was selected for wet etching because it is free of metal ions and has a very low oxide etch rate. Figure 1 shows the SEM photograph of a Si tip array. TMAH concentration below $20 \%$ often generated undesired hillocks and a higher nonuniformity in the emitter arrays. It has been reported that at $95^{\circ} \mathrm{C}$, the silicon etch rate generally drops as the TMAH concentration increases above $20 \% .^{11}$ The etch rate of $\langle 100\rangle$ silicon in our experiment dropped from 1.27 to $0.5 \mu \mathrm{m} / \mathrm{min}$ as the TMAH concentration was increased from $20 \%$ to $40 \%$ at $90^{\circ} \mathrm{C}$. Typical Si tips have a pyramidal shape

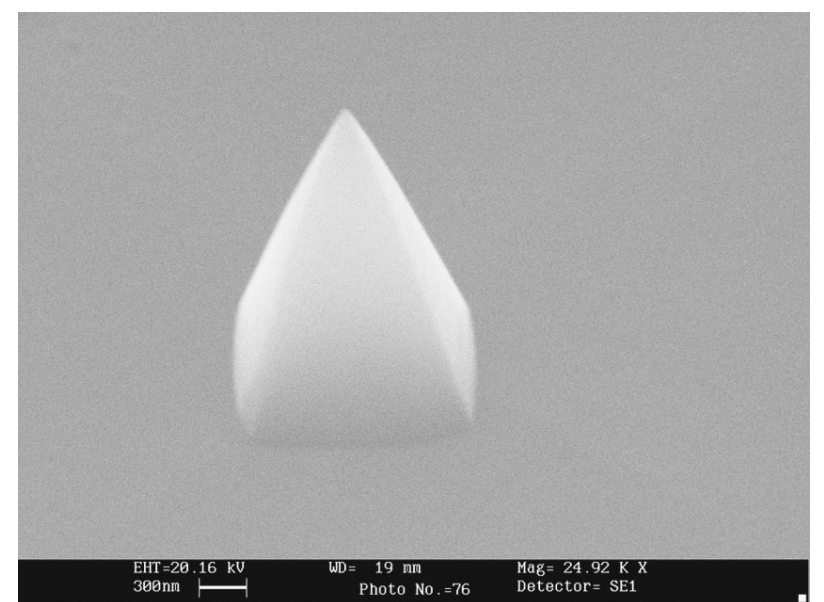

FIG. 2. Closer view of a single silicon tip. The scale bar corresponds to 300 nm.

with a $1.44 \mu \mathrm{m}$ base and $2.20 \mu \mathrm{m}$ height, as seen in the SEM photograph in Fig. 2. The pyramidal tip is bound by four facets with the edges of the pyramid base always aligned to the $\{310\}$ planes, irrespective of how the initial oxide mask pattern is aligned. The aspect ratio of the pyramid was found to be 1.53 by measuring the base and height of the pyramids located at different regions of the array. Based on the orientation and the tilt angle, we determined that the facets of the pyramid were in the group of $\{311\}$ crystal planes. Hence the etch rate of these planes was 2.16 times faster than that of the (100) plane. The difference in etch rates may be related to the surface atom density and activation energy. The etching nonuniformity was determined as the ratio of the root-meansquare differences of each pyramid base versus the average base width. By using $40 \%$ (weight) TMAH at $90^{\circ} \mathrm{C}, 1.4 \%$ nonuniformity was achieved for the etched base. This unique self-terminating etch process produces pyramidal shaped tips suitable for field emission. On completion of silicon undercutting, the oxide caps fell off, and the etching was terminated. The tips were further sharpened by thermal oxidation. Figure 3 shows the tips sharpened by different oxidation times. It can be seen that a 30 min wet oxidation, which grows a $0.25 \mu \mathrm{m}$ oxide layer, does not sharpen the tip appreciably. For a 60 min wet oxidation, about $0.5 \mu \mathrm{m}$ oxide layer was grown, and the tip was sharpened significantly. Further oxidation did not have much effect on the sharpness of the tip but reduced the height of the tips, as can be seen from the tips sharpened by 2 and $4 \mathrm{~h}$ oxidation steps. The radius of the Si tips was reduced from $100 \mathrm{~nm}$ after etching to about $20 \mathrm{~nm}$ after $1 \mathrm{~h}$ oxidation as obtained from SEM measurement. Oxidation sharpening changed the tip shape from pyramid to concave, which is favorable for field emission applications since its field enhancement factor is larger compared to that of the normal pyramidal shaped tips. ${ }^{12}$

\section{B. Diamond deposition}

Unlike nucleation on a flat surface, diamond nucleation on delicate sharp tips is difficult. Nucleation and growth of single diamond particle on metal tips have been achieved in 


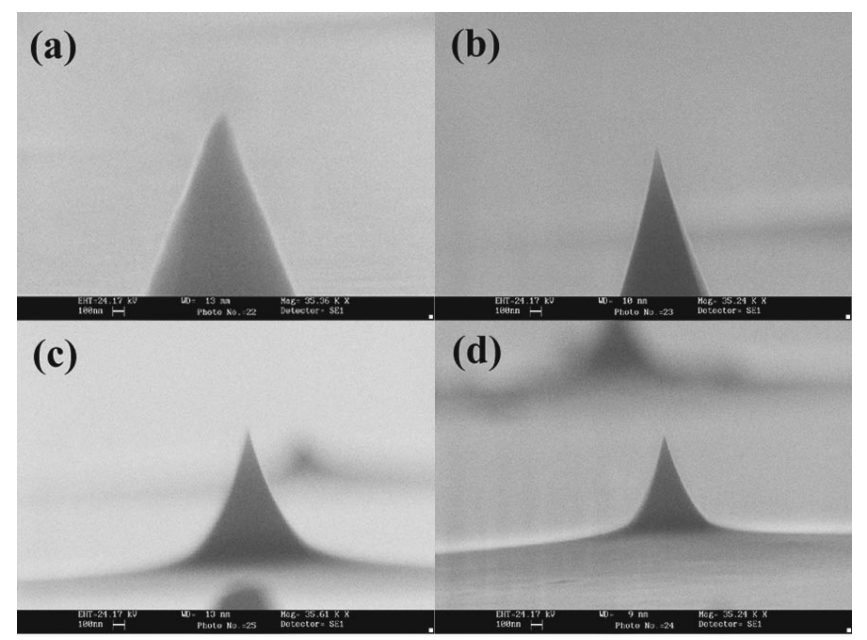

FIG. 3. Silicon tips sharpened by oxidation at various times: (a) $30 \mathrm{~min}$, (b) $60 \mathrm{~min}$, (c) $120 \mathrm{~min}$, (d) $240 \mathrm{~min}$. The scale bar is $100 \mathrm{~nm}$.

our laboratory. ${ }^{13}$ By combining the nanodiamond slurry and negative bias, we have grown a thin uniform diamond coating on to the tips, which can be clearly seen from the SEM photograph in Fig. 4. A typical Raman spectrum of the film is shown in Fig. 5, where the $1332 \mathrm{~cm}^{-1}$ peak corresponds to diamond. The broad band spectrum centered around $1550 \mathrm{~cm}^{-1}$ indicates the presence of $s p^{2}$ carbon. The same nucleation method is also applied to selectively coat the tips for the gated field emitter array shown in Fig. 6(a). A closer view of a diamond coated emitter tip shown in Fig. 6(b) reveals selective nucleation, which was achieved using a photoresist mask that was removed in acetone after the nucleation. Under the growth conditions used, a diamond film of approximately $100 \mathrm{~nm}$ was grown in $30 \mathrm{~min}$ as measured by the SEM from a cleaved flat sample. After diamond coating, the radius of the emitter tips increased from $20 \mathrm{~nm}$ to approximately $50 \mathrm{~nm}$, but the tips maintained the original profile.

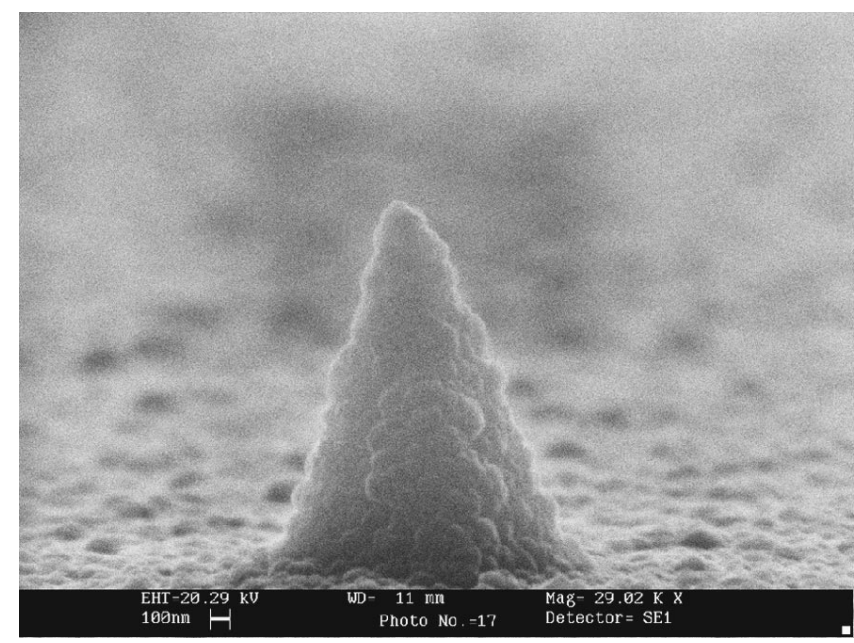

FIG. 4. Diamond coated field emitter tip. The scale bar is $100 \mathrm{~nm}$.

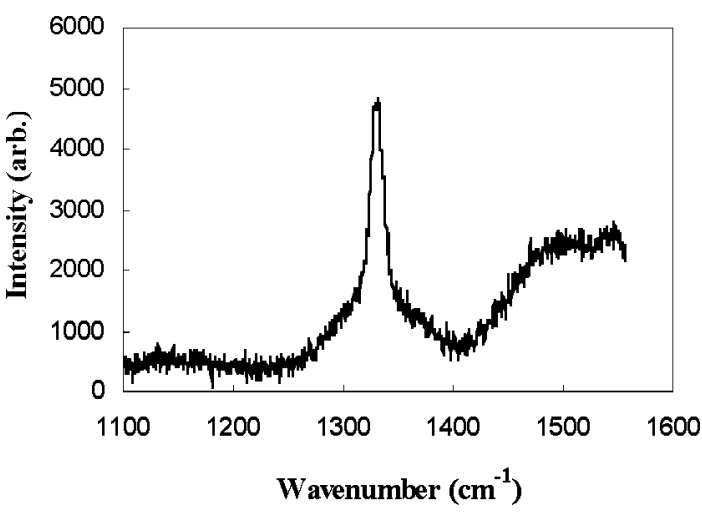

FIG. 5. Typical Raman spectrum of the diamond film.

\section{I- $V$ measurement}

The emission properties of diamond coated emitter arrays were tested in a vacuum chamber at $10^{-6}$ Torr. The diode configuration was used for samples without gate. The anode was placed directly on top of the emitter array, separated by

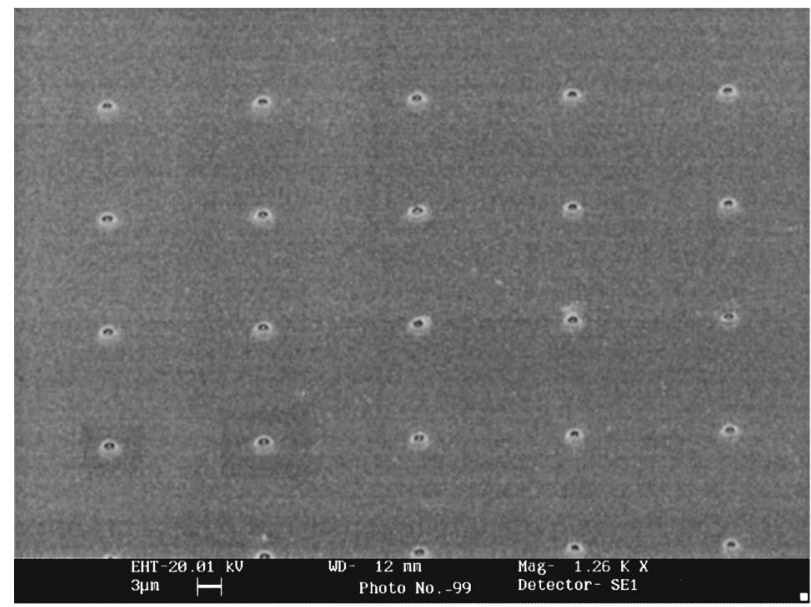

(a)

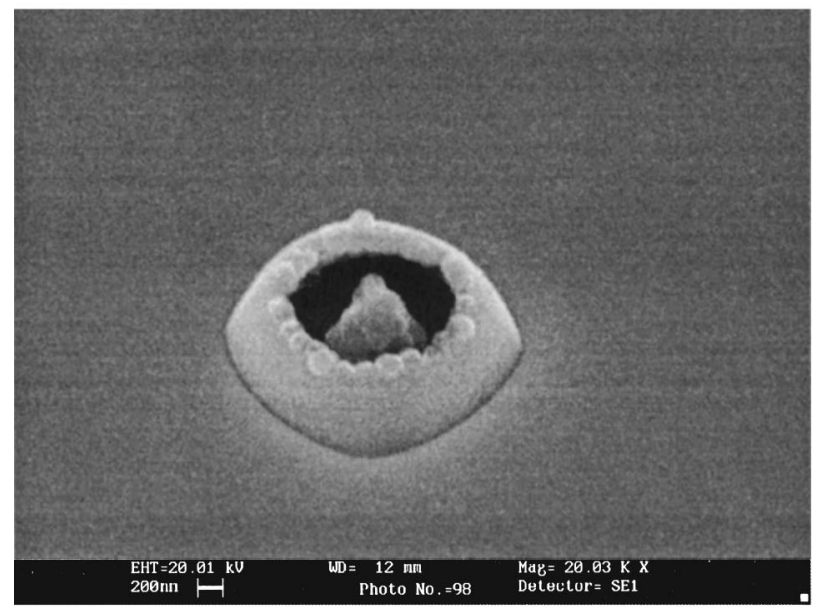

(b)

FIG. 6. Diamond coated field emitter with self-aligned gate: (a) an array, (b) a magnified view of a single emitter. 


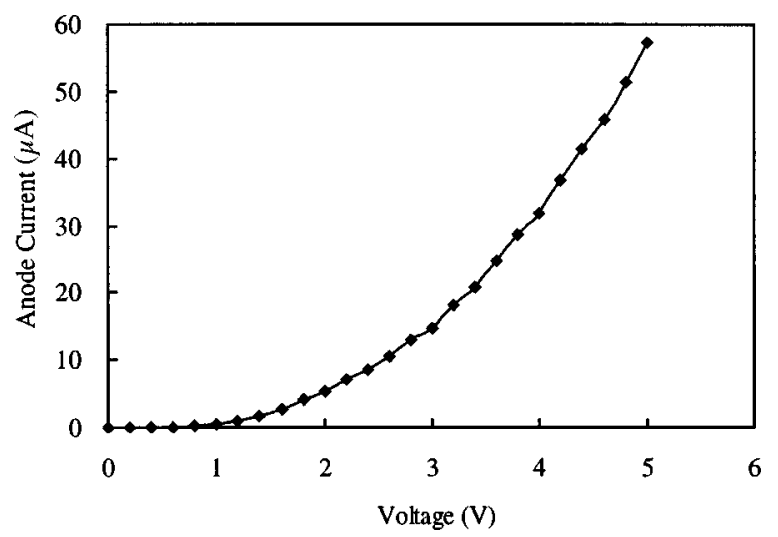

FIG. 7. $I-V$ characteristics of the diamond coated field emitter arrays without gate.

a thermal oxide of $2 \mu \mathrm{m}$ total thickness. The breakdown field of the oxide layer was measured to be higher than 200 $\mathrm{V} / \mu \mathrm{m}$. The total number of emitters on the test sample was 400 , formed in a $20 \times 20$ array. Figure 7 shows the result of emission $I-V$ measurements. The turn-on voltage of the emitter, defined as the voltage where anode current exceeds $10 \mathrm{nA}$, is about $0.2 \mathrm{~V}$. The emission current reached $57 \mu \mathrm{A}$ at an anode voltage of $5 \mathrm{~V}$. If we assume that all the emissions occurred uniformly at the emitter tips, the average current density, for a tip radius of $50 \mathrm{~nm}$, could reach $800 \mathrm{~A} / \mathrm{cm}^{2}$. The high emission current shows the potential of using diamond emitters as an electron source. Similar results were obtained from a nitrogen doped diamond pyramid. ${ }^{14}$ We attribute the low turn-on voltage and high emission current of the array to the thin diamond coating and the field enhancement from the sharp tip. The $I-V$ characteristics of the gated emitter array are shown in Fig. 8. The turn-on voltage is about $40 \mathrm{~V}$. The emission current reached $3.4 \mu \mathrm{A}$ at a gate voltage of $80 \mathrm{~V}$.

The tunneling current from the tips can be described by the Fowler-Nordheim $(\mathrm{F}-\mathrm{N})$ equation: ${ }^{15}$

$$
J=\frac{A E^{2}}{\phi t^{2}(y)} \exp \left(-B \frac{\phi^{3 / 2}}{E} v(y)\right),
$$

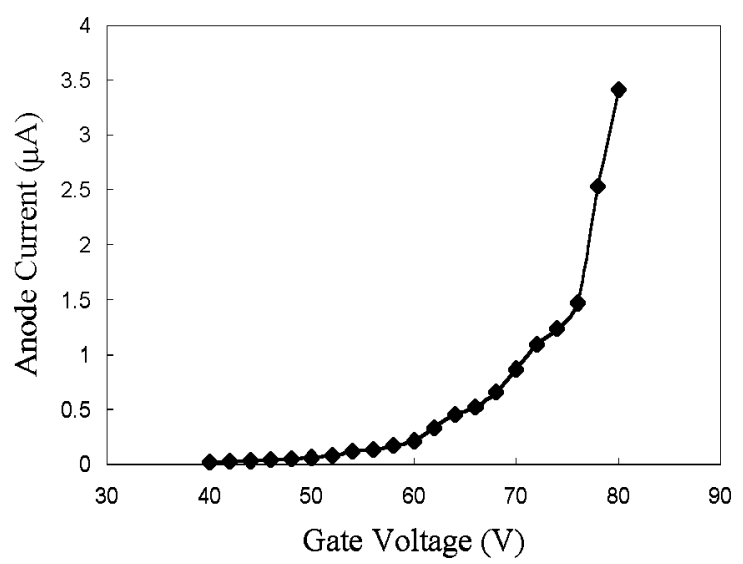

FIG. 8. $I-V$ characteristics of diamond coated field emitter array with selfaligned gate.

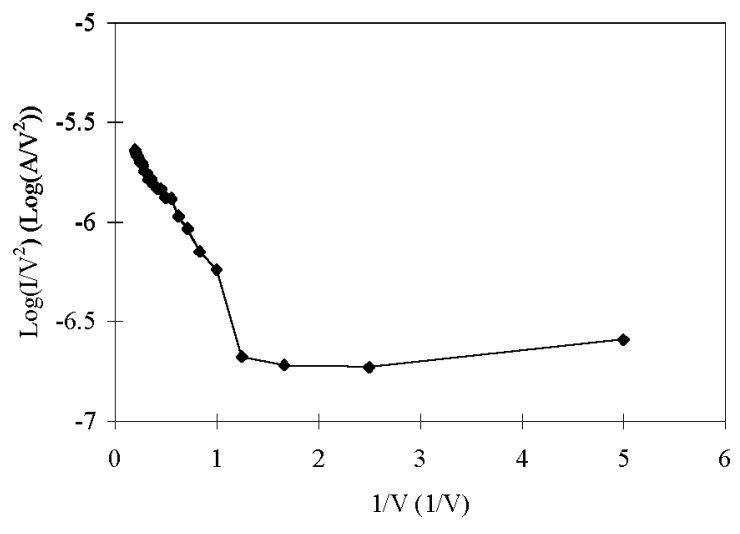

FIG. 9. Fowler-Nordheim plot of diamond coated field emitter array without gate.

where $\quad A=1.54 \times 10^{-6}, \quad B=6.87 \times 10^{7}, \quad y=3.79$ $\times 10^{-4} E^{1 / 2} / \phi, E$ is the electric field at the cathode, and $\phi$ is the work function of the cathode material. The functions $v(y)$ and $t(y)$ at the range of the field encountered in our device can be approximated by the following values:

$$
t^{2}(y)=1.1
$$

and

$$
v(y)=0.95-y^{2} .
$$

The emission current and the applied voltage are related to the current density and the electric field by $J=I / \alpha$ and $E=\beta V$, where $\alpha$ is the emitting area and $\beta$ is the field conversion factor considering both the separation of the electrodes and the field enhancement due to sharp tips. The $\mathrm{F}-\mathrm{N}$ equation can be described, in terms of current $I$ and voltage $V$, as:

$$
I=a V^{2} \exp \left(-\frac{b}{V}\right)
$$

where

$$
\begin{aligned}
& a=\frac{\alpha A \beta^{2}}{1.1 \phi} \exp \left(\frac{\left(1.44 \times 10^{-7}\right) B}{\phi^{1 / 2}}\right), \\
& b=\frac{0.95 B \phi^{3 / 2}}{\beta} .
\end{aligned}
$$

From Eq. (3), the $\mathrm{F}-\mathrm{N}$ plot, $\log \left(I / V^{2}\right)$ vs $(1 / V)$ will give a straight line of slope $-b \propto \phi^{3 / 2}$, where $\phi$ is the work function of diamond. Figures 9 and 10 show the $\mathrm{F}-\mathrm{N}$ plots for emitter arrays without and with gate, respectively. The emission current at a high field shows a typical $\mathrm{F}-\mathrm{N}$ characteristic. From the simulation result of Zheng, ${ }^{12}$ the field conversion factor $\beta_{\text {gated }}$ of our gated diamond field emitters is found to be $1.76 \times 10^{5} \mathrm{~cm}^{-1}$ for a $50 \mathrm{~nm}$ tip radius. Using this value of $\beta_{\text {gated }}$, the work function of the diamond coating was calculated to be $1.08 \mathrm{eV}$, which is lower than that reported for the DLC coating. ${ }^{9}$ The flattening of the $\mathrm{F}-\mathrm{N}$ plot at a low field shows the presence of surface leakage current.

At a low field, the slope $b$ in Eq. (5) is free of the effect of the field. At a higher field, however, the slope of the $\mathrm{F}-\mathrm{N}$ 


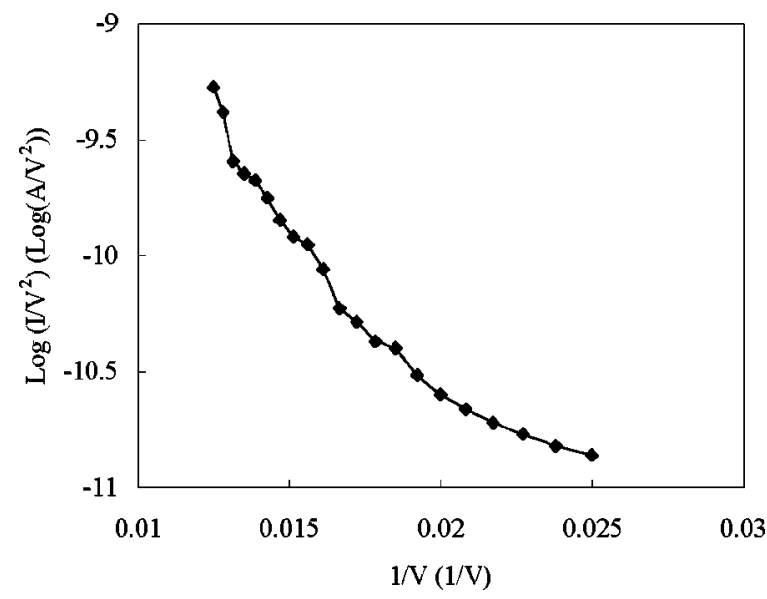

FIG. 10. Fowler-Nordheim plot of diamond coated field emitter array with self-aligned gate.

plot will decrease due to increase in $\beta$. This is evident from the $\mathrm{F}-\mathrm{N}$ plot of emitter arrays without gate, for which the slope $b$ is smaller than that of the gated emitter array. Similar results were also reported by Okano and Kwon for diamond film. ${ }^{14,16}$ Using $\phi=1.08 \mathrm{eV}$, we have calculated the field conversion factor $\beta_{\text {ungated }}$ for emitter without gate to be $4.33 \times 10^{7} \mathrm{~cm}^{-1}$, which is approximately two orders of magnitude higher than $\beta_{\text {gated }}$. Hence, it is not surprising to observe a low turn-on voltage and high emission current for the ungated device. Similarly, we conclude that since $\beta_{\text {gated }}$ is low, the gated emitters require a higher operating voltage to produce equivalent emission current.

\section{CONCLUSIONS}

Diamond coated field emitter arrays, with and without a self-aligned gate, have been successfully fabricated. Sharp silicon tips with less than $1.4 \%$ nonuniformity were obtained by wet chemical etching in $40 \%$ TMAH. The etching process was found self-terminating at a $\{311\}$ group of crystal facets on a $\langle 100\rangle$ silicon substrate. A $100 \mathrm{~nm}$ thick diamond film was uniformly coated onto the tip arrays using negative bias assisted growth after nucleation with nanocrystalline diamond slurry. The effective work function of the diamond coating was estimated to be $1.08 \mathrm{eV}$. The turn-on voltages of diamond coated field emitter arrays with and without gate were 40 and $0.2 \mathrm{~V}$, respectively. The difference is attributed to the high field enhancement in the ungated device. Our fabrication technique is suitable for low voltage vacuum electronic devices and microsensors based on diamond field emitter arrays.

\section{ACKNOWLEDGMENTS}

The research at Old Dominion University was supported by grants from National Aeronautics and Space Administration (Grant No. NAG-1-1995) and Varian Associates. The authors acknowledge many useful discussions with Dr. S. K. Kahng and Dr. G. A. Rooks.

${ }^{1}$ C. A. Spindt, J. Appl. Phys. 39, 3504 (1968).

${ }^{2}$ K. Betsui, Technical Digest of the 4th International Vacuum Microelectronics Conference, Nagahama, Japan, 1991, p. 26 (unpublished).

${ }^{3}$ N. A. Fox, W. N. Wang, T. J. Davis, J. W. Steeds, and P. W. May, Appl. Phys. Lett. 71, 2337 (1997).

${ }^{4} \mathrm{Ph}$. Niedermann, W. Hänni, N. Blanc, R. Christoph, and J. Burger, J. Vac. Sci. Technol. A 14, 1233 (1996).

${ }^{5}$ S. Lee, B. K. Ju, Y. H. Lee, D. Jeon, and M. H. Oh, J. Vac. Sci. Technol. B 15, 425 (1997).

${ }^{6}$ K. H. Park, S. Lee, K. H. Song, J. I. Park, K. J. Park, S. Y. Han, S. J. Na, N. Y. Lee, and K. H. Koh, J. Vac. Sci. Technol. B 16, 724 (1998).

${ }^{7}$ V. V. Zhirnov, E. I. Givargizov, A. V. Kandidov, B. V. Seleznev, and A. N. Alimova, J. Vac. Sci. Technol. B 15, 446 (1997).

${ }^{8}$ H. C. Cheng, T. K. Ku, B. B. Hsien, S. H. Chen, S. Y. Leu, C. C. Wang, C. F. Chen, I. J. Hsieh, and J. C. M. Huang, Jpn. J. Appl. Phys., Part 1 34, 6926 (1995).

${ }^{9}$ F. Y. Chuang, C. Y. Sun, H. F. Cheng, W. C. Wang, C. M. Huang, and I. N. Lin, Technical Digest of the 9th International Vacuum Microelectronics Conference, St. Petersburg, Russia, 7-12 July 1996, p. 339.

${ }^{10}$ C. C. Zhu, H. Guan, W. Liu, and T. Y. Li, J. Vac. Sci. Technol. B 15, 1682 (1997).

${ }^{11}$ Lj. Ristic, H. Hughes, and F. Shemansky, Sensor Technology and Devices (Artech House, Boston, 1994), p. 49.

${ }^{12}$ C. Zheng and L. Tong, IEEE Trans. Electron Devices 40, 448 (1993).

${ }^{13}$ S. Albin, J. Zheng, J. Cooper, W. Fu, and A. C. Lavarias, Appl. Phys. Lett. 71, 2848 (1997).

${ }^{14}$ K. Okano, T. Yamada, H. Ishihara, S. Koizumi, and J. Itoh, Appl. Phys. Lett. 70, 2201 (1997).

${ }^{15}$ C. A. Spindt, I. Brodie, L. Humphrey, and E. R. Westerberg, J. Appl. Phys. 47, 5248 (1976).

${ }^{16}$ S. J. Kwon, Y. H. Shin, D. M. Aslam, and J. D. Lee, J. Vac. Sci. Technol. B 16, 712 (1998). 